\title{
Fractional Euler-Bernoulli beams: theory, numerical study and experimental validation
}

\author{
W. Sumelka*, T. Blaszczyk ${ }^{* *}$, C. Liebold ${ }^{* * *}$ \\ *Poznan University of Technology, Institute of Structural Engineering, \\ Piotrowo 5 street, 60-969 Poznan Poland \\ wojciech.sumelka@put.poznan.pl \\ ${ }^{* *}$ Czestochowa University of Technology, Institute of Mathematics \\ al. Armii Krajowej 21, 42-201 Czestochowa, Poland \\ tomasz.blaszczyk@im.pcz.pl \\ *** Berlin University of Technology, Institute of Mechanics \\ Einsteinufer 5, 10587 Berlin, Germany \\ christian.liebold@tu-berlin.de
}

Keywords: Euler-Bernoulli beams, fractional calculus, non-local models

\begin{abstract}
In this paper the classical EULER-BERNOULLI beam (CEBB) theory is reformulated utilising fractional calculus. Such generalisation is called fractional Euler-Bernoulli beams (FEBB) and results in non-local spatial description. The parameters of the model are identified based on AFM experiments concerning bending rigidities of micro-beams made of the polymer SU-8. In experiments both force as well as deflection data were recorded revealing significant size effect with respect to outer dimensions of the specimens. Special attention is also focused on the proper numerical solution of obtained fractional differential equation.
\end{abstract}

\section{Introduction}

A quantitative understanding of a size effect in engineering materials is of great importance in the design phase of micro- and nano-electromechanical systems (MEMS and NEMS), and as a technique of homogenisation regarding materials with real micro-structure. A size effect is reflected, for example, in a stiffer elastic response to external forces on small scales. This has been recognised in several experiments on metals and polymers, for example on copper (FLECK et al. 1994 [17]), silver (MA and ClARKe 1995 [24]), zinc oxide (STAN et al. 2007 [35]), lead (CUENOT et al. 2004 [12]), carbon nanotubes (SALVETAT et al. 1999 [33]), epoxy (CHONG 2002 [10]) and polypropylene (MCFARLAND and COLTON 2005 [25]). Actually, a physical reasoning for the origin of a size-dependent material behaviour, such as non-negligible complex interactions of molecular chains in polymers (NIKOLOV et al. 2007 [27]), the rearrangement of atoms or molecules near the surface, the influence of the grain-size in polycystals (SMYSHLYAEV and FLECK 1996 [34]) and long-ranging influences from dislocations, voids or some inner micro- or nano-structure, is manifold and poorly understood especially in combined effects. For the reason that conventional continuum theory is unable to predict size effect, different 
Wojciech Sumelka, Tomasz Blaszczyk, Christian Liebold - Compiled on 2015/02/06 at 01:34:59

non-conventional continua are proposed in literature, like non-local theories (PEDDIESON et al. 2003 [31], ERINGEN 2010 [16]), strain-gradient theories (TOUPIN 1962 [39], MINDLIN and EsHEL 1968 [26]), micropolar theories (ERINGEN 1966 [15], NOWACKI 1972 [28]) or theories of material surfaces (GURTIN \& MURDOCH 1975 [18]).

In addition to these, the fractional calculus is assumed to be a promising candidate for modelling scale-dependent material behaviour too, by using a concept of intrinsic fractal structures to describe a potential disordered material's micro-structure (cf. CARPINTERI 1994 [8]). For detailed descriptions see KlimeK (2001) [20], VAZAQuez (2004) [41], Lazopoulos (2006) [21], Di PAOla et al. (2009) [13], Atanackovic \& Stankovic (2009) [1], CARPinteri et el. (2011) [9] or DrapaCA \& SivalOGANATHAN (2012) [14]. The formulation of fractional elasticity stated here defines spatial derivatives of arbitrary order including the positive features - in contrast to the specified non-conventional continua - (1) to introduce a smaller number of additional material parameters, (2) to fit into the general framework of classical continuum mechanics and (3) to possess clear physical interpretation (e.g. regarding to the intrinsic length scale).

In Sect. 2.1, we start with a description of a generalised continuum mechanical framework including the specific formalism of fractional calculus proposed in SUMELKA 2014 [36]. More precisely, we define the gradient of the motion of a body, i.e. the deformation gradient, with the help of an differentiation operator a of real order. Having this, strain and stress measures, as well as a formulation of the conservation of momentum will be derived according to conventional kinematics of small deformations. In Sect. 2.2 the classical EULER-BERNOULLI assumptions will be utilised to define a displacement field valid for bending of slender beams. By applying equilibrium considerations to an infinitesimal beam element, the fractional EULER-BERNOULLI differential equation will be presented as the basis for the numerical study. Section 3.1 clarifies the boundary conditions for the model in accordance with the experimental realisation. We will investigate the bending behaviour of micro-beams with rectangular cross-sections that are clamped on one side and loaded at the other by a single concentrated force. The computational algorithm, explained in more detail in Sect. 3.2, will use a one-dimensional mesh of equidistantly distributed calculation points along the beam's axis. In between these nodes, the fractional EULER-BERNOULLI differential equation will be solved including additional conditions for the transition of moments and forces between the sub-elements of the model. However, the operator for the differentiation of real order requires an additional step of extended integration over the neighbourhood of the relevant point to collect and weigh the non-local information of the body. The step of integration will be implemented using the fractional trapezoidal scheme ODIBAT (2006) [29], BLASZCZYK et al. (2013) [6] and SUMELKA \& BLASZCZYK (2014) [37]. All these preparations allow us to build a stiffness matrix of the system, to be solved by the LU decomposition method. The benchmark numerical results will be presented in Sect. 3.3 for beams of different (thicknesses/lengths) and different fractional parameters will be applied to optimise the results to fit to the experiments.

Finally, in Sect. 4 with the help of an Atomic Force Microscope (AFM) force as well as deflection data of micro-beams made of the polymer material SU-8 the fractional beam model will be identified. The experimental approach and the measured data will be explained to emphasise that the bending stiffness of the SU-8 structures revealed a size effect on the thickness and the length of the samples. 


\section{Fractional Euler-Bernoulli beams}

\subsection{Small strain fractional elasticity}

There are many ways to generalise continuum mechanics in terms of fractional calculus, as mentioned in KlimeK (2001) [20], VAzaquez (2004) [41], LAzopoulos (2006) [21], Di PAOla et al. (2009) [13], ATANACKOVIC \& Stankovic (2009) [1], CARPinteri et el. (2011) [9] or DraPACA \& SivaLOGANATHAN (2012) [14]. Herein we follow the concept presented in SUMELKA (2014) [36]. Because the FEBB theory results from putting specific restrictions on general small strain fractional elasticity, we start with a short introduction to the latter one - for details cf. SUMELKA (2014) [36], SUMELKA \& BLASZCZYK (2014) [37].

The concept of fractional continua discussed in SUMELKA (2014) [36] is based on fractional deformation gradients concept, namely

$$
\underset{X}{\tilde{\mathbf{F}}}(\mathbf{X}, t)=\ell_{X}^{\alpha-1}{\underset{X}{D}}^{\alpha} \phi(\mathbf{X}, t), \quad \text { or } \quad \underset{X}{\tilde{F}} a A=\ell_{A}^{\alpha-1}{\underset{X}{D}}_{D^{\alpha}}^{\alpha} \phi_{a} \mathbf{e}_{a} \otimes \mathbf{E}_{A},
$$

and

$$
\underset{x}{\tilde{\mathbf{F}}}(\mathbf{x}, t)=\ell_{x}^{\alpha-1}{ }_{x}^{\alpha} \varphi(\mathbf{x}, t), \quad \text { or } \quad \underset{x}{\tilde{F}} A a=\ell_{a}^{\alpha-1}{ }_{x_{a}}^{D^{\alpha}} \varphi_{A} \mathbf{E}_{A} \otimes \mathbf{e}_{a},
$$

where $\underset{X}{\tilde{\mathbf{F}}}$ and $\underset{x}{\tilde{\mathbf{F}}}$ are fractional deformation gradients in material and spatial descriptions, respectively, $\ell_{X}$ and $\ell_{x}$ are corresponding length scales, $\phi$ defines the regular motion of the material body while $\varphi$ its inverse. The fractional differential operator $D^{\alpha}$ is defined as the Riesz-Caputo (RC) fractional derivative

$$
{ }_{a}^{\mathrm{RC}} D_{b}^{\alpha} f(t)=\frac{1}{2} \frac{\Gamma(2-\alpha)}{\Gamma(2)}\left({ }_{a}^{\mathrm{C}} D_{t}^{\alpha} f(t)+(-1)^{n}{ }_{t}^{\mathrm{C}} D_{b}^{\alpha} f(t)\right)
$$

where $\alpha>0$ denotes the real order of the derivative, $D$ denotes 'derivative' (RC stands for RIESZCAPUTO), $a, t, b$ are so called terminals, $\Gamma$ is the EulER gamma function, ${ }_{a}^{\mathrm{C}} D_{t}^{\alpha} f(t),{ }_{t}^{\mathrm{C}} D_{b}^{\alpha} f(t)$ are left and right CAPUTO's fractional derivatives, respectively PODLUBNY (1999) [32], KILBAS ET AL. (2006) [19], LESZCZYNSKI (2011) [22]. From the definition of the RC derivative by Eq. (3) it is clear, that fractional deformation gradients given by Eqs (1) and (2) are non-local (each component of $\underset{X}{\tilde{\mathbf{F}}}$ and $\underset{x}{\tilde{\mathbf{F}}}$ governs the information from the surrounding, described by terminals $a$ and $b$ ). In consequence, other related measures of deformation (e.g. strain tensor) which are obtained through the relations analogous as in classical case, are also non-local. To conclude, fractional kinematics is controlled by new material parameters: length scale, order of derivation and type of fractional derivative OLIVEIRA \& MACHADO (2014) [30] - they should be identified for specific material.

Thus, the finite fractional strains are obtained from the difference in scalar products in actual and reference configurations (cf. Fig. 1)

$$
\begin{aligned}
& \mathbf{E}=\frac{1}{2}\left(\stackrel{\mathbf{F}}{T}^{T} \stackrel{\diamond}{\mathbf{F}}-\mathbf{I}\right), \quad \text { or } \quad E_{A B}=\frac{1}{2}\left(\stackrel{\diamond}{F}_{A a}^{T} \stackrel{\diamond}{F}_{a B}-I_{A B}\right) \mathbf{E}_{A} \otimes \mathbf{E}_{B}
\end{aligned}
$$

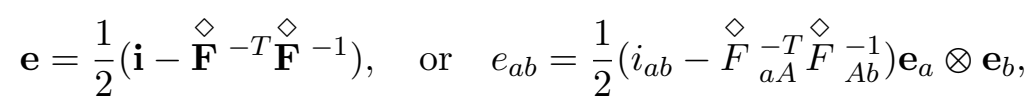

where $\mathbf{E}$ is the classical GREEN-LAGRANGE strain tensor or its fractional counterpart (symmetric), e is the classical EULER-ALMANSI strain tensor or its fractional counterpart (symmetric), and $\overrightarrow{\mathbf{F}}$ can be replaced with $\mathbf{F}$ or $\underset{X}{\tilde{\mathbf{F}}}$ or $\underset{x}{\tilde{\mathbf{F}}}$ or $\stackrel{\alpha}{\mathbf{F}}$ (cf. Fig. 1). 


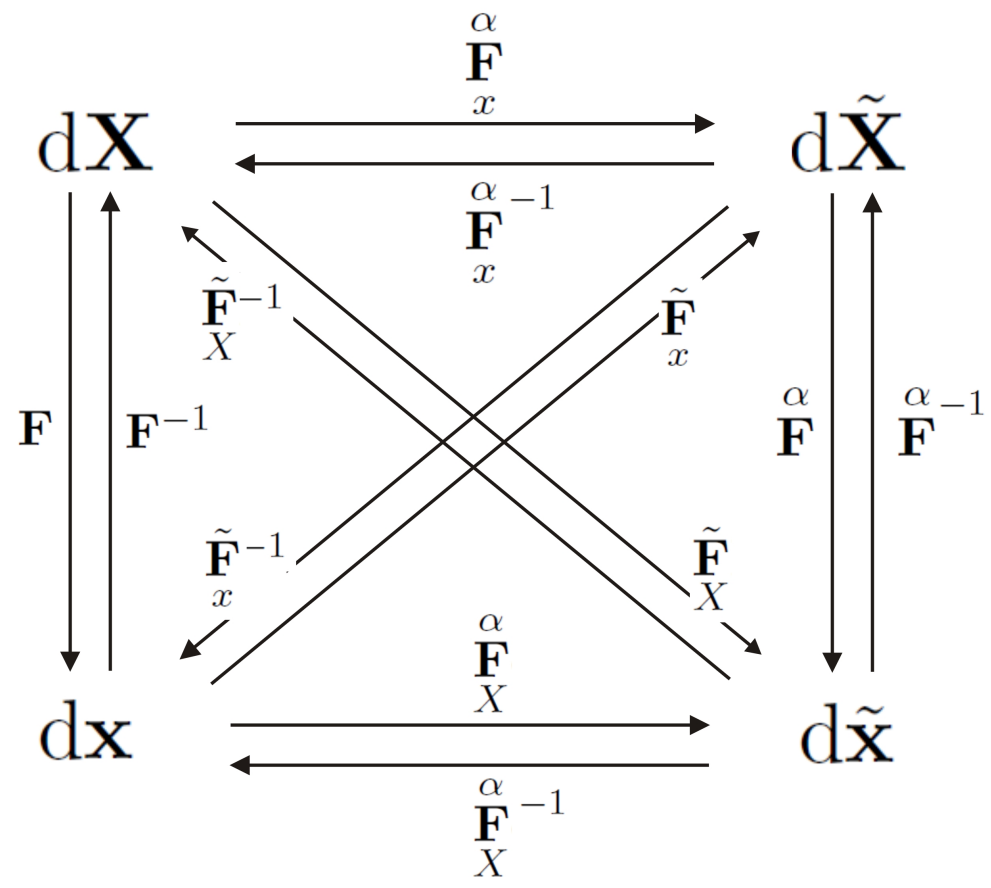

Figure 1: The relationship between material and spatial line elements with their fractional counterparts $\stackrel{\alpha}{\mathbf{F}}=\underset{X}{\tilde{\mathbf{F}}} \mathbf{F}^{-1} \underset{x}{\tilde{\mathbf{F}}}-1, \underset{x}{\mathbf{F}}=\underset{x}{\tilde{\mathbf{F}} \mathbf{F}}$ and $\underset{X}{\stackrel{\alpha}{\mathbf{F}}}=\underset{X}{\tilde{\mathbf{F}}} \mathbf{F}^{-1}$

An infinitesimal fractional strain is obtained as in the classical set-up by considering the relationship between fractional strains and fractional displacement gradients tensors and omitting higher order terms in obtained relations. Hence, infinitesimal fractional CAUCHY strain tensor is defined as (assuming that $\ell=\ell_{X}=\ell_{x}$ )

$$
\stackrel{\grave{\varepsilon}}{=} \frac{1}{2}\left[\operatorname{Grad} \tilde{\mathbf{U}}+\underset{X}{\operatorname{Grad}} \tilde{\mathbf{U}}^{T}\right]=\frac{1}{2}\left[\underset{x}{\operatorname{grad} \tilde{\mathbf{u}}}+\underset{x}{\operatorname{grad}} \tilde{\mathbf{u}}^{T}\right]
$$

where

$$
\underset{X}{\operatorname{Grad}} \underset{X}{\tilde{\mathbf{U}}}=\underset{X}{\tilde{\mathbf{F}}}-\mathbf{I}, \quad \text { or } \quad \ell^{\alpha-1}{ }_{X_{A}}^{{ }^{\alpha}} U_{a}=\left(\underset{X}{\tilde{F}} a A-I_{a A}\right) \mathbf{e}_{a} \otimes \mathbf{E}_{A},
$$

and

$$
\operatorname{grad} \tilde{\mathbf{u}}=\mathbf{i}-\underset{x}{\tilde{\mathbf{F}}}, \quad \text { or } \quad \ell^{\alpha-1}{ }_{x_{a}}^{D^{\alpha}} u_{A}=\left(i_{A a}-\underset{x}{\tilde{F}} A a\right) \mathbf{E}_{A} \otimes \mathbf{e}_{a} .
$$

In the above equations $\mathbf{U}$ and $\mathbf{u}$ denote material and spatial displacement.

It is clear that for the fractional kinematics appropriate fractional kinetics should be defined SUMELKA et al. (2014) [38]. It can be shown that fractional stress field $\tilde{\boldsymbol{\sigma}}$ satisfies analogous relations as in the classical formulation. Assuming that the conservation of mass holds, hence

$$
\rho_{0} \mathrm{~d} V=\tilde{\rho_{0}} \mathrm{~d} \tilde{V}=\rho \mathrm{d} v=\tilde{\rho} \mathrm{d} \tilde{v},
$$

or shortly

$$
\stackrel{\diamond}{\rho_{0}}=\stackrel{\diamond}{J} \rho
$$

where $\rho_{0}\left(\tilde{\rho_{0}}\right)$ is the reference mass density (fractional counterpart), $\rho(\tilde{\rho})$ is spatial mass density (frac-

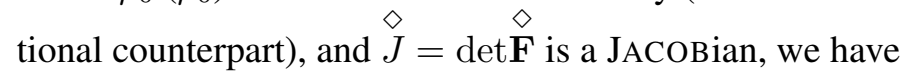

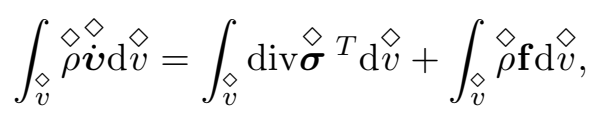


where $\boldsymbol{v}$ is a velocity, and $\mathbf{f}$ is a body force per unit mass, so the local form is

$$
\operatorname{div} \stackrel{\vartheta}{\boldsymbol{\sigma}}^{T}+\stackrel{\diamond}{\rho} \mathbf{f}=\stackrel{\diamond}{\rho} \dot{\boldsymbol{v}}
$$

or in the absence of inertia forces

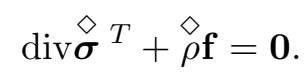

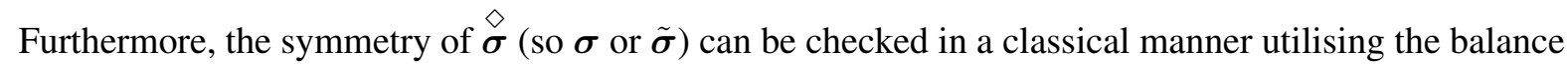
of moment of momentum. The result given by Eq. (12) gives justification and is analogical for the one postulated in ATANACKOVIC \& STANKOVIC (2009) [1] for similar small strain elasticity generalisation. Finally, the small strain fractional elasticity is governed by (for purpose the denotation $(\tilde{\cdot})$ is omitted)

$$
\left\{\begin{array}{l}
\sigma_{i j, j}+b_{i}=0, \\
\stackrel{\vartheta}{\varepsilon}_{i j}=\frac{1}{2} \ell^{\alpha-1}\left({ }_{X_{j}}{ }^{\alpha} u_{i}+{\stackrel{D}{X_{i}}}^{\alpha} u_{j}\right)=\frac{1}{2} \ell^{\alpha-1}\left(u_{i, \breve{j}}+u_{j, \breve{i}}\right), \\
\sigma_{i j}=\mathcal{L}_{i j k l}^{e} \stackrel{\stackrel{\varepsilon}{\varepsilon}}{k l}, \\
U_{i}=\check{U}_{i}, \quad \mathbf{X} \in \Omega_{U}, \\
\sigma_{i j} n_{j}=\check{t}_{i}, \quad \mathbf{X} \in \Omega_{\sigma}, \\
\Omega_{U} \cap \Omega_{\sigma}=\emptyset \quad \text { and } \quad \Omega_{U} \cup \Omega_{\sigma}=\Omega .
\end{array}\right.
$$

In the above equation we have denoted: $\mathbf{b}$ is the body force, $\mathcal{L}^{e}$ is the stiffness tensor, $\Omega_{U}$ and $\Omega_{\sigma}$ are parts of boundary $\Omega$ where the displacements and the tractions are applied, respectively. It is clear that the classical (local) solution appears as a special case of the proposed generalisation when $\alpha=1$.

\subsection{Governing equations}

Governing equations for the FEBB are obtained under the analogous assumptions as in a classical case (for classical case cf. BAUCHAU \& CRAIG (2009) [3]). Without loss of generality we consider the effect of extension and bending (other concepts like SAINT-VENANT assumptions associated with torsion can be incorporated by analogy).

The classical ad hoc assumptions for kinematics hold, namely:

Assumption 1 The cross-section is infinitely rigid in its own plane,

Assumption 2 The cross-section of a beam remains plane after deformation,

Assumption 3 The cross-section remains normal to the deformed axis of the beam.

Within the above assumptions we can express the 3D displacements in terms of the 1D beam displacements (direction 1 coincides with a beam axis):

$$
\begin{aligned}
& u_{1}\left(x_{1}, x_{2}, x_{3}\right)=\bar{u}_{1}\left(x_{1}\right)+x_{3} \Phi_{2}\left(x_{1}\right)-x_{2} \Phi_{3}\left(x_{1}\right), \\
& u_{2}\left(x_{1}, x_{2}, x_{3}\right)=\bar{u}_{2}\left(x_{1}\right) \\
& u_{3}\left(x_{1}, x_{2}, x_{3}\right)=\bar{u}_{3}\left(x_{1}\right) .
\end{aligned}
$$

In Eqs (15) we have assumed the following sign convention: the rigid body translations of the crosssection $\bar{u}_{1}\left(x_{1}\right), \bar{u}_{2}\left(x_{1}\right)$, and $\bar{u}_{3}\left(x_{1}\right)$ are positive in the positive direction of the coordinate axis, respectively; the rigid body rotations of the cross-section $\Phi_{2}\left(x_{1}\right)=-\frac{\mathrm{d} \bar{u}_{3}\left(x_{1}\right)}{\mathrm{d} x_{1}}$ and $\Phi_{3}\left(x_{1}\right)=\frac{\mathrm{d} \bar{u}_{2}\left(x_{1}\right)}{\mathrm{d} x_{1}}$ are positive keeping the right-hand rule. 
Because finally the experimental set-up considers bending in (1,3)-plane we reduce the set of Eqs (15) to

$$
\begin{aligned}
& u_{1}\left(x_{1}, x_{2}, x_{3}\right)=-x_{3} \frac{\mathrm{d} \bar{u}_{3}\left(x_{1}\right)}{\mathrm{d} x_{1}}, \\
& u_{2}\left(x_{1}, x_{2}, x_{3}\right)=0 \\
& u_{3}\left(x_{1}, x_{2}, x_{3}\right)=\bar{u}_{3}\left(x_{1}\right) .
\end{aligned}
$$

Applying the displacement relations Eqs (16) to the definition of fractional CAUCHY strain Eq. (6) we observe that the only non-zero element is

$$
\stackrel{\diamond}{\varepsilon}_{11}=-x_{3} \ell^{\alpha-1}{\underset{X}{1}}^{\alpha}\left(\frac{\mathrm{d} \bar{u}_{3}\left(x_{1}\right)}{\mathrm{d} x_{1}}\right)=z \ell^{\alpha-1} \breve{\kappa}_{2}=-z \ell^{\alpha-1} w,{ }_{1 \breve{1}},
$$

so

$$
\sigma_{11}=-z \ell^{\alpha-1} E w_{1 \breve{1}}
$$

where $z=x_{3}, E$ is YounG's modulus, $w=\bar{u}_{3}\left(x_{1}\right)$ and $\breve{\kappa}_{2}\left(x_{1}\right)=-w,_{1 \breve{1}}$. It is clear that the transverse shear stress due to flexure cannot be obtained in this way due to the third EULER-BERNOULLI assumption - it is determined from equilibrium considerations as in the classical set-up (we further denote it by $\left.\sigma_{13}^{*}\right)$.

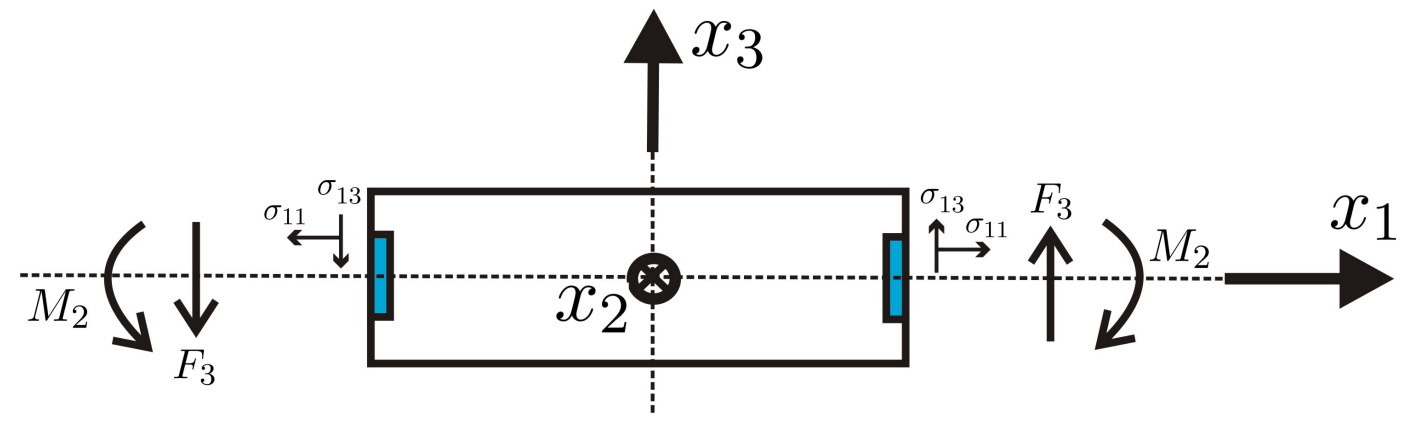

Figure 2: The sectional stress resultants.

Next, following Fig. 2 the kinetic variables, called the sectional stress resultants, are

$$
M_{2}=\int_{A} \sigma_{11} x_{3} \mathrm{~d} A=-w,{ }_{1 \breve{1}} \int_{A} z^{2} \ell^{\alpha-1} E \mathrm{~d} A,
$$

and

$$
F_{3}=\int_{A} \sigma_{13}^{*} \mathrm{~d} A
$$

Up to this point we have three unknowns: displacement $\bar{u}_{3}$, (fractional) strain $\breve{\kappa}_{2}$ of the beam, and stress resultant $M_{1}$. Thus far, we have obtained two equations which combine them, namely:

- strain-displacement relation $\breve{\kappa}_{2}\left(x_{1}\right)=-D_{X_{1}}{ }^{\alpha}\left(\frac{\mathrm{d} \bar{u}_{3}\left(x_{1}\right)}{\mathrm{d} x_{1}}\right)$, and

- constitutive relation $M_{2}=\breve{\kappa}_{2} \int_{A} z^{2} \ell^{\alpha-1} E \mathrm{~d} A$. 
The missing last one is derived using equilibrium considerations, so called NEwTONian method.

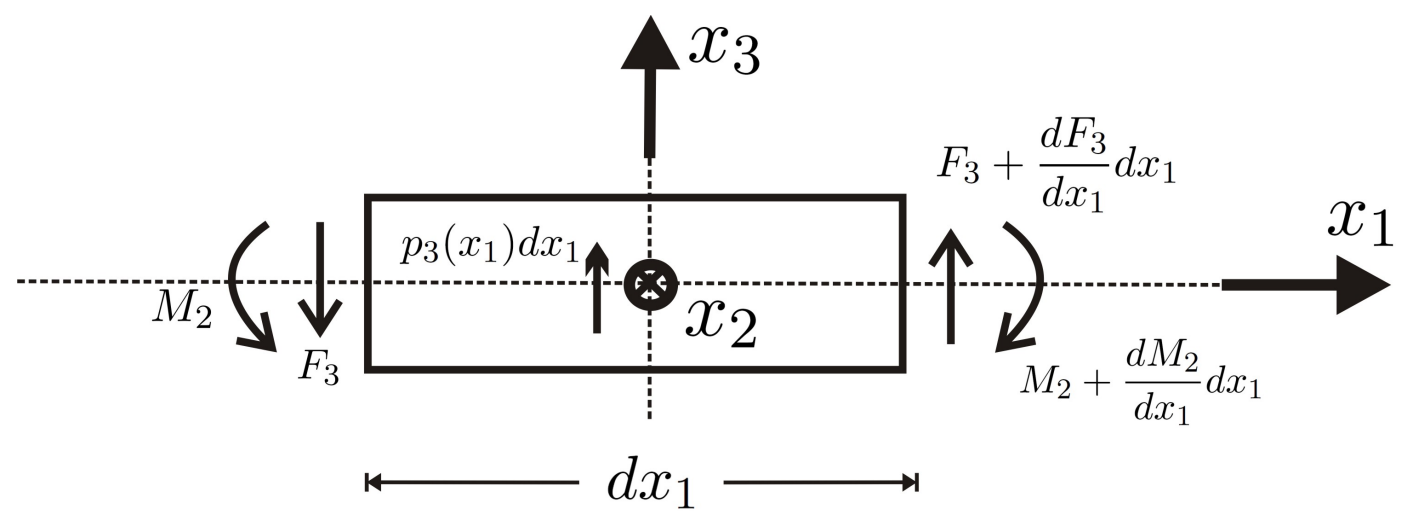

Figure 3: Free body diagram for the transverse shear forces and bending moments.

Thus, in the absence of distributed moments through the beam axis, following the free body diagram shown in Fig. (3), we have the following relations

$$
\frac{\mathrm{d} F_{3}}{\mathrm{~d} x_{1}}=-p_{3}\left(x_{1}\right)
$$

and

$$
\frac{\mathrm{d} M_{2}}{\mathrm{~d} x_{1}}-F_{3}=0
$$

where $p_{3}\left(x_{1}\right)$ denotes the distributed load. Next, by taking a derivative of Eq. (22) then introducing Eq. (21), the bending moment equilibrium equation is

$$
\frac{\mathrm{d}^{2} M_{2}}{\mathrm{~d} x_{1}^{2}}=-p_{3}\left(x_{1}\right)
$$

which defines the third lacking equation, for the full description of beam bending in the plane (1,3) (in the absence of distributed moments through the beam axis).

Finally, combining Eq. (19) and Eq. (23) we obtain a single equation in terms of $w$

$$
\ell^{\alpha-1} E I w,{ }_{1111}=p_{3}\left(x_{1}\right),
$$

where $I=\int_{A} z^{2} \mathrm{~d} A$. When order of fractional continua is taken $\alpha=1$ classical solution is recovered

$$
\ell^{0} E I w, 1111=E I \frac{\mathrm{d}^{4} w}{\mathrm{~d} x_{1}^{4}}=p_{3}\left(x_{1}\right)
$$

\section{Numerical study}

\subsection{Bending test}

The real physical conditions in micro-beam bending tests, discussed in Sect. 4, correspond to a cantilever beam model, loaded at the free end by a point load $P$ - cf. Fig. 4. 


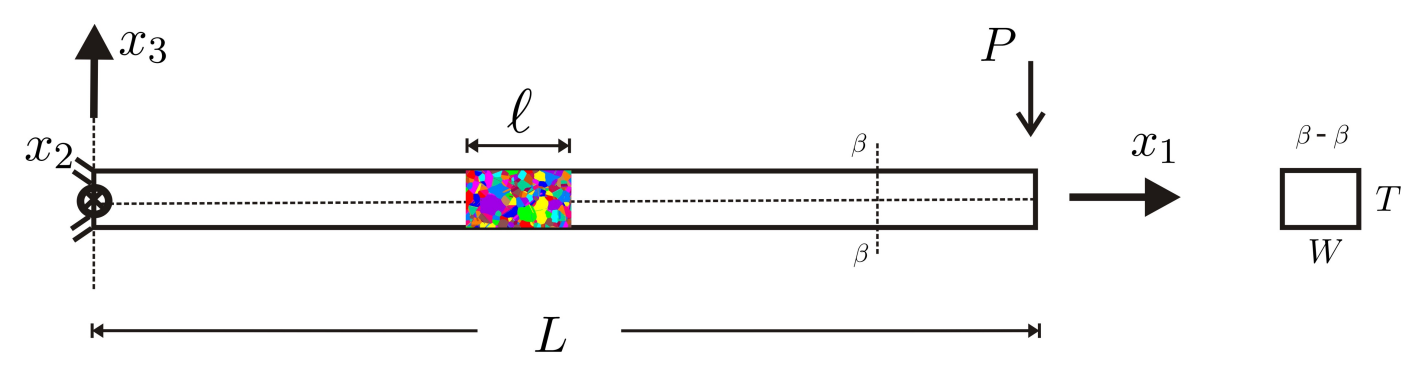

Figure 4: Computational model of a micro-beam bending test.

For such a configuration resultant moment is known, namely

$$
M_{2}\left(x_{1}\right)=P L-P x_{1},
$$

thus Eq. (23) is satisfied. Therefore the problem is governed by (cf. Eq. (19))

$$
\left\{\begin{array}{l}
-D_{X_{1}}^{\alpha}\left(\frac{\mathrm{d} w}{\mathrm{~d} x_{1}}\right) \ell^{\alpha-1} E I=P L-P x_{1} \\
w\left(x_{1}=0\right)=0 \\
\frac{\mathrm{d} w}{\mathrm{~d} x_{1}}\left(x_{1}=0\right)=0
\end{array}\right.
$$

where $I=\frac{W T^{3}}{12}$.

\subsection{Computational algorithm}

In this section we present a numerical scheme for the problem (27). We introduce the homogeneous grid of nodes (see Fig. 5). We see that additional fictitious nodes $x_{1}^{-m}, \ldots, x_{1}^{-1}$ and $x_{1}^{N+1}, \ldots, x_{1}^{N+m}$ placed outside the domain $\left[x_{1}^{0}, x_{1}^{N}\right]$ are introduced. We denote a value of the beam deflection at the node $x_{1}^{i}$ as $w\left(x_{1}^{i}\right)=w_{i}$. Next, by analogy as in CIESIELSKI \& LESZCZYNSKI (2006) [11], SUMELKA \& BLASZCZYK (2014) [37], we assume that for all fictitious nodes on the left the beam deflections are $w_{-m}=w_{-m+1}=\ldots=w_{-1}=w_{0}$ and on the right the beam deflections are $w_{N+m}=w_{N+m-1}=$ $\ldots=w_{N+1}=w_{N}$.

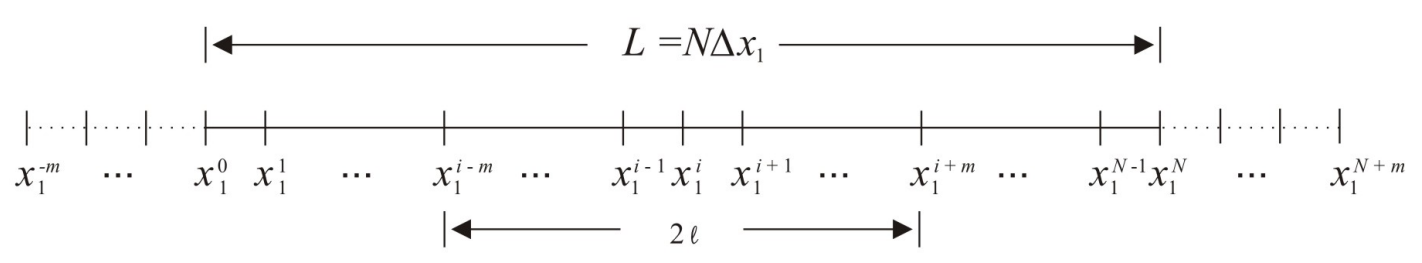

Figure 5: Spatial discretization for micro-beam.

The problem of numerical solutions of equations containing simultaneously the left and right fractional derivatives has recently been drawing the attention of many authors ASAD et al. (2014) [2], 
Blaszczy \& Ciesielski (2014) [5], Blaszczy (2015) [4], Cresson et al. (2013) [7], XU \& AGRAWAL (2014) [42]. In this paper we use a scheme which is based on the fractional trapezoidal rule Odibat (2006) [29], BlAsZCZYK et al. (2013) [6] and SumELKa \& BLASZCZYK (2014) [37]. The left CAPUTO derivatives at nodes $x_{1}^{i}$ is approximated by the formula

$$
\begin{aligned}
\left.\mathrm{C}_{x_{1}^{i-m}} D_{x_{1}}^{\alpha}\left(\frac{\mathrm{d} w}{\mathrm{~d} x_{1}}\right)\right|_{x_{1}=x_{1}^{i}} & =\frac{1}{\Gamma(1-\alpha)} \int_{x_{1}^{i-m}}^{x_{1}^{i}} \frac{w^{\prime \prime}(\tau) \mathrm{d} \tau}{\left(x_{1}^{i}-\tau\right)^{\alpha}} \\
& \cong \frac{\left(\Delta x_{1}\right)^{1-\alpha}}{\Gamma(3-\alpha)}\left\{\left[(m-1)^{2-\alpha}-(m+\alpha-2) m^{1-\alpha}\right] w^{\prime \prime}\left(x_{1}^{i-m}\right)+w^{\prime \prime}\left(x_{1}^{i}\right)\right. \\
& \left.+\sum_{j=i-m+1}^{i-1}\left[(i-j-1)^{2-\alpha}-2(i-j)^{2-\alpha}+(i-j+1)^{2-\alpha}\right] w^{\prime \prime}\left(x_{1}^{j}\right)\right\},
\end{aligned}
$$

where $(\cdot)^{\prime \prime}$ denotes a second derivative with respect to $x_{1}$. Using the central difference formula for the second order derivatives occurring in (28) we finally obtain the approximation of the left CAPUTO derivative

$$
\left.{ }_{x_{1}^{i-m}}^{\mathrm{C}} D_{x_{1}}^{\alpha} w\right|_{x_{1}=x_{1}^{i}} \cong \frac{\left(\Delta x_{1}\right)^{-1-\alpha}}{\Gamma(3-\alpha)} \sum_{j=i-m-1}^{i+1} w_{j} v_{1}(i, j),
$$

where

$v_{1}(i, j)=\left\{\begin{array}{ll}(m-1)^{2-\alpha}-(m+\alpha-2) m^{1-\alpha} & \text { for } j=i-m-1 \\ (m-2)^{2-\alpha}-4(m-1)^{2-\alpha} & \text { for } j=i-m \\ +2(m+\alpha-2) m^{1-\alpha}+m^{2-\alpha} & \\ -2(m-2)^{2-\alpha}+5(m-1)^{2-\alpha} & \text { for } j=i-m+1 \wedge m=2 \\ -(m+\alpha-2) m^{1-\alpha}-2 m^{2-\alpha}+1 & \\ (m-3)^{2-\alpha}-4(m-2)^{2-\alpha}+6(m-1)^{2-\alpha} & \text { for } j=i-m+1 \wedge m>2 \\ -(m+\alpha-2) m^{1-\alpha}-m^{2-\alpha} & \\ (i-j-2)^{2-\alpha}-4(i-j-1)^{2-\alpha}+6(i-j)^{2-\alpha} & \\ -4(i-j+1)^{2-\alpha}+(i-j+2)^{2-\alpha} & \text { for } j=i-m+2, \ldots, i-2 \wedge m>3 \\ 6-4 \cdot 2^{2-\alpha}-3^{2-\alpha} & \text { for } j=i-1 \wedge m>2 \\ 2^{2-\alpha}-4 & \text { for } j=i \\ 1 & \text { for } j=i+1 \\ 0 & \text { otherwise }\end{array}\right.$. 
We determine the discrete form of the right fractional CAPUTO derivative in a similar way

$$
\begin{aligned}
\left.{ }_{x_{1}}^{\mathrm{C}} D_{x_{1}^{i+m}}^{\alpha}\left(\frac{\mathrm{d} w}{\mathrm{~d} x_{1}}\right)\right|_{x_{1}=x_{1}^{i}} & =\frac{-1}{\Gamma(1-\alpha)} \int_{x_{1}^{i}}^{x_{1}^{i+m}} \frac{w^{\prime \prime}(\tau) \mathrm{d} \tau}{\left(\tau-x_{1}^{i}\right)^{\alpha}} \\
& \cong \frac{-\left(\Delta x_{1}\right)^{1-\alpha}}{\Gamma(3-\alpha)}\left\{\left[(m-1)^{2-\alpha}-(m+\alpha-2) m^{1-\alpha}\right] w^{\prime \prime}\left(x_{1}^{i+m}\right)+w^{\prime \prime}\left(x_{1}^{i}\right)\right. \\
& \left.+\sum_{j=i-1}^{i+m+1}\left[(j-i-1)^{2-\alpha}-2(j-i)^{2-\alpha}+(j-i+1)^{2-\alpha}\right] w^{\prime \prime}\left(x_{1}^{j}\right)\right\} \\
& \cong \frac{-\left(\Delta x_{1}\right)^{-1-\alpha}}{\Gamma(3-\alpha)} \sum_{j=i-1}^{i+m+1} w_{j} v_{2}(i, j)
\end{aligned}
$$

where

$$
v_{2}(i, j)= \begin{cases}(m-1)^{2-\alpha}-(m+\alpha-2) m^{1-\alpha} & \text { for } j=i+m+1 \\ (m-2)^{2-\alpha}-4(m-1)^{2-\alpha} & \text { for } j=i+m \\ +2(m+\alpha-2) m^{1-\alpha}+m^{2-\alpha} & \text { for } j=i+m-1 \wedge m=2 \\ -2(m-2)^{2-\alpha}+5(m-1)^{2-\alpha} & \\ -(m+\alpha-2) m^{1-\alpha}-2 m^{2-\alpha}+1 & \text { for } j=i+m-1 \wedge m>2 \\ (m-3)^{2-\alpha}-4(m-2)^{2-\alpha}+6(m-1)^{2-\alpha} & \\ -(m+\alpha-2) m^{1-\alpha}-m^{2-\alpha} & \text { for } j=i+2, \ldots, i+m-2 \wedge m>3 \\ (j-i-2)^{2-\alpha}-4(j-i-1)^{2-\alpha}+6(j-i)^{2-\alpha} & \text { for } j=i+1 \wedge m>2 \\ -4(j-i+1)^{2-\alpha}+(j-i+2)^{2-\alpha} & \text { for } j=i \\ 6-4 \cdot 2^{2-\alpha}-3^{2-\alpha} & \text { for } j=i-1 \\ 2^{2-\alpha}-4 & \text { otherwise } \\ 1 & \end{cases}
$$

Finally, we present the discrete form of the considered problem (27). For calculation of values $w_{0}, w_{1}, \ldots, w_{N}$ we need to solve the system of $N+1$ linear equations. For every grid node $x_{1}^{i}$, where $i=0, \ldots, N$, we can write the following equations

$$
\left\{\begin{array}{l}
w_{0}=0 \\
w_{1}=0 \\
\sum_{j=i-m-1}^{i+1} w_{j} v_{1}(i, j)+\sum_{j=i-1}^{i+m+1} w_{j} v_{2}(i, j)=\frac{P\left(L-i \Delta x_{1}\right)}{\beta}
\end{array}\right.
$$

where

$$
\beta=-\frac{\ell^{\alpha-1} E I}{\left(\Delta x_{1}\right)^{1+\alpha} \Gamma(3-\alpha)} .
$$

The parameter $m$, in above formulae, represents the number of spatial nodes for which the fractional derivatives, Eq. (28) and (31), are calculated. The relation between the parameter $m$, length scale $\ell$ and step size $\Delta x_{1}$ is expressed by: $\Delta x_{1}=\frac{\ell}{m}$. Thus, for larger values of $\ell, m$ should also be increased appropriately, because the convergence of solution, represented in Eq. (33), is in relation to $\Delta x_{1}$. 


\subsection{Benchmark results}

As in the CEBB theory, also in its fractional non-local version, the information about the cross section is introduced through the moment of inertia $I$ only. In this sense, the FEBB theory makes it possible to analyse the non-local effects through beam length $L$. In the following series of benchmark results we consider the influence of length scale $\ell$ and order of fractional continua $\alpha$ on cantilever beam bending test results.

On the basis of the numerical scheme presented in the previous section we implemented a computer program in Maple and carried out computational simulations for different values of parameters $\alpha, \ell$ and $m$. We applied the LU decomposition method in order to numerically solve the system of equations (33). In all presented benchmark examples we assumed $P=1 \mathrm{~N}, E=1 \mathrm{~Pa}, L=1 \mathrm{~m}, W=T=\frac{1}{10} L$ and $\Delta x_{1}=0.001 \mathrm{~m}$. We denote by $w(L, \alpha)$ a value of a beam deflection at the loaded end $w\left(x_{1}^{N}\right)$ for fixed $\alpha \in\{0.4,0.5,0.6,0.7,0.8,0.9,0.99\}$ and by $w(L, 1)$ a value of a beam deflection at the loaded end $w\left(x_{1}^{N}\right)$ for classical local i.e. $\alpha=1$. In Fig. 6 the numerical results are presented. Two cases are considered: (i) the interval of fractional differentiation $2 \ell$ is smaller (or equal) than the smallest beam dimension $T$, and (ii) $2 \ell$ is smaller (or equal) then beam length $L$.
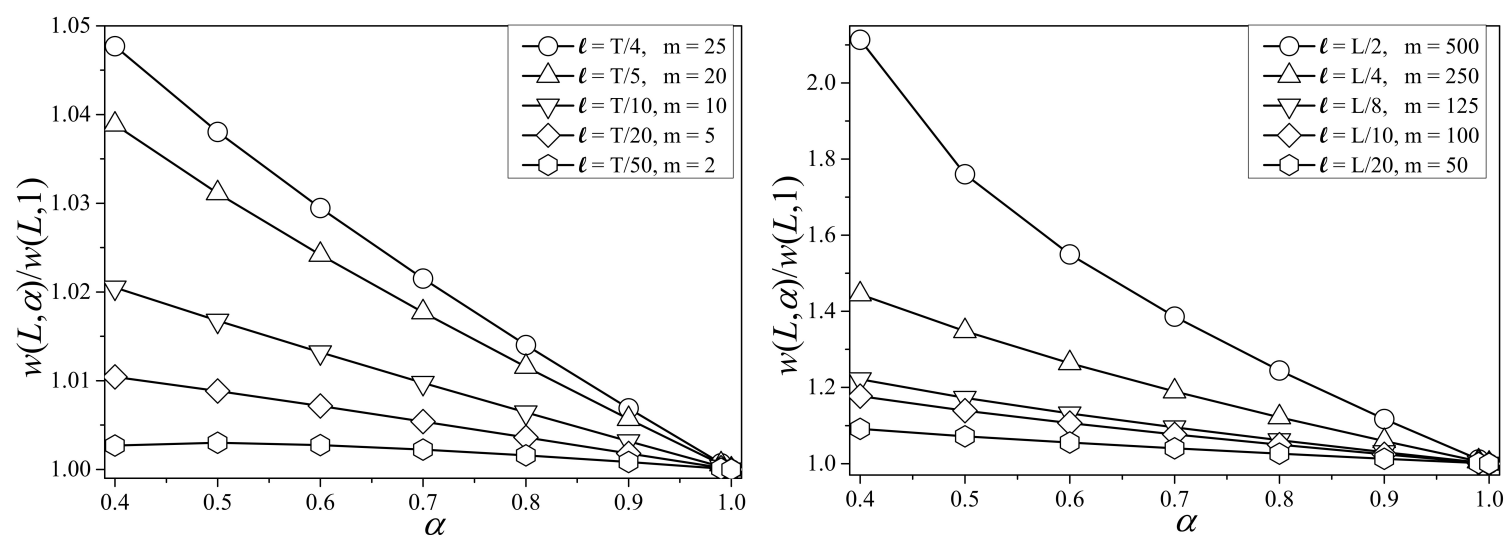

Figure 6: The relation between the normalised cantilever beam deflection at loaded end vs. order of fractional continua $\alpha$ for different length scales $\ell$.Left: case (i). Right: case (ii).

We observe that when length scale $\ell$ increases in comparison to beam length $L$, the non-local effects are more vivid. If $\ell$ approaches zero and/or $\alpha$ one, the FEBB reduces to the CEBB model. It is also clearly seen that for smaller values of $\alpha$ the difference between a classical and fractional result increases. We can conclude that both $\ell$ and $\alpha$ control the stiffness of the fractional beam - this result is crucial concerning model identification in the next Sect. 4.

\section{Experimental validation}

\subsection{Experimental set-up}

Deflection and force data from real micro-beam bending tests were recorded with an off-axis laserreflective Multiview-1000 AFM-stage from Nanonics Imaging Ltd. The system is composed of a flat scanner, consisting of a fine thread driven by piezo-elements with high-voltage power supply and a detection device working with four Photo-Sensitive Diods (PSD) interconnected as a WHEATSTONE 
bridge to monitor deflections of a laser-beam path. The laser is reflected in an obtuse angle from a fixed AFM-cantilever such that the system directly monitors its deflections $w_{\mathrm{c}}$, when deformed by the piezo uplift (referred to as separation $z$ ). With the knowledge of the well calibrated spring constant of the AFM-cantilever of $k_{\mathrm{c}}=31.4 \mathrm{~N} \mathrm{~m}^{-1}$, we were able to convert the PSD-signal into force $P=k_{\mathrm{c}} w_{\mathrm{c}}$ (cf., Fig. 7). The calibration process used here is described in more detail in VARENBERG et al. (2005) [40] and was performed using a precise silicon normal that was provided by the PTB (Physikalisch Technische Bundesanstalt - Braunschweig). The AFM-cantilever is a custom-built cylindrical and curved cantilever made of glass, having a tip radius of about $20 \mathrm{~nm}$. In micro-beam bending tests it can be assumed that the pure AFM-data consist of a combined signal of the deflection of the AFM-cantilever and the micro-beam's deflection $w$ in the following manner: $z=w+w_{\mathrm{c}}$. Hence, we were able to separate the deflection data of a micro-beam from the pure AFM-data in combination with the corresponding load, cf. Fig. 7.
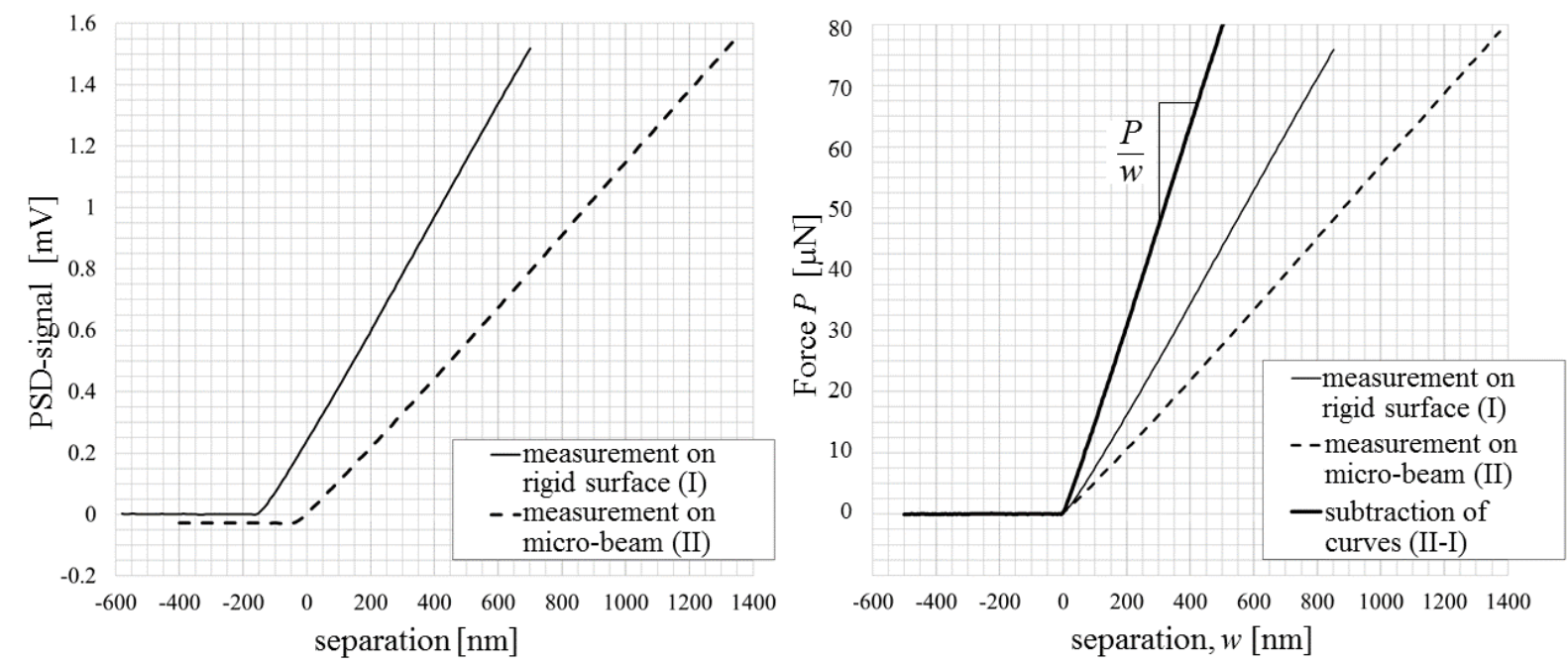

Figure 7: Some exemplary original PSD-data (left) as well as shifted and converted data (right).

The ratio $P / w$ is called bending stiffness with the classical relationship to the elastic modulus $E$, width $W$, length $L$ and thickness $T$

$$
E=\frac{4 L^{3}}{W T^{3}} \frac{P}{w}
$$

when the CEBB theory and a rectangular cross-section of the beam are assumed. The length and width of the samples have been measured in an optical microscope with a magnification of 500 times, whereas the thickness was taken to be the mean value from two different optical determination systems and values of a Scanning Electron Microscope (SEM). Thereby, the lengths were specified between the fixation of the cantilever on the solid glass support and the force application point of the AFM-tip, which could be varied.

SU-8 is known as the photo-resist Nano ${ }^{\mathrm{TM}}$-SU-8 of the company MicroChem, used in the micro-system technology as described in LORENZ et al. (1997) [23]. Manufacturing of the samples was carried out in the following steps:

- A 4-inch silicon wafer was coated with a thin metal film as a barrier layer. 


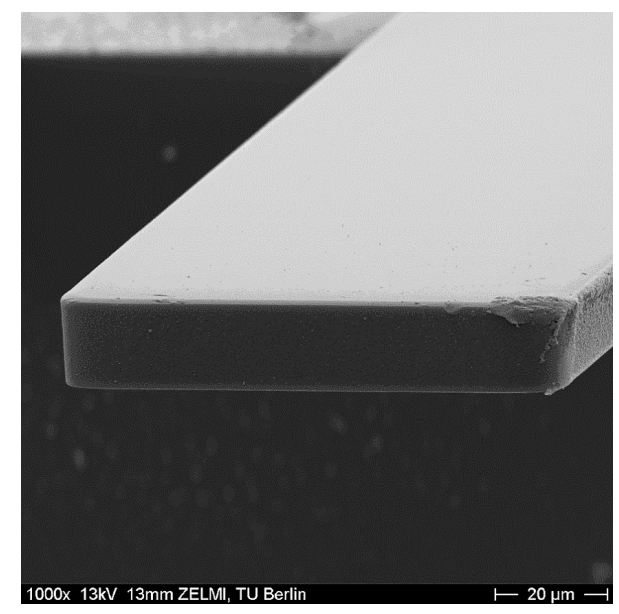

Figure 8: An exemplary SEM picture of the free end of a tested micro-beam made of SU-8.

- First, the viscous SU-8 resist was dissolved in solvents. A conventional spin-coating machine was applied to disperse the resist on the metal-coated silicon wafer. The rotational speed of the coating machine determined the thickness of the homogeneous layer (between 8-40 microns).

- The solvent evaporated in the rotary process in large part and the remaining part was evaporated in a subsequent drying process at temperatures of $60^{\circ} \mathrm{C}-95^{\circ} \mathrm{C}$, whereby the material finally received its rigidity.

- Structuring was achieved by a Laser Direct Imager (LDI). After exposure to light an additional heat treatment was carried out at $60^{\circ} \mathrm{C}-95^{\circ} \mathrm{C}$ to assist the chemical reaction of illumination.

- By using a proper developer, the regions of exposed SU-8 were dissolved from unexposed regions.

- Followed by a chemical etch process, which does not attack the SU-8, the thin metal film on the silicon wafer was dissolved and the micro-beam structures were finally peeled off.

- In a last step the micro-beam structures were glued on a support made of glass and fixed on an AFM sample holder.

Samples of different lengths and widths have been produced in different thicknesses $(T=8.4 \mu \mathrm{m}$ and $14.4 \mu \mathrm{m})$ at the Fraunhofer Institute for Reliability and Microintegration, Berlin, and were tested with the AFM-technique according to Eq. 35. 


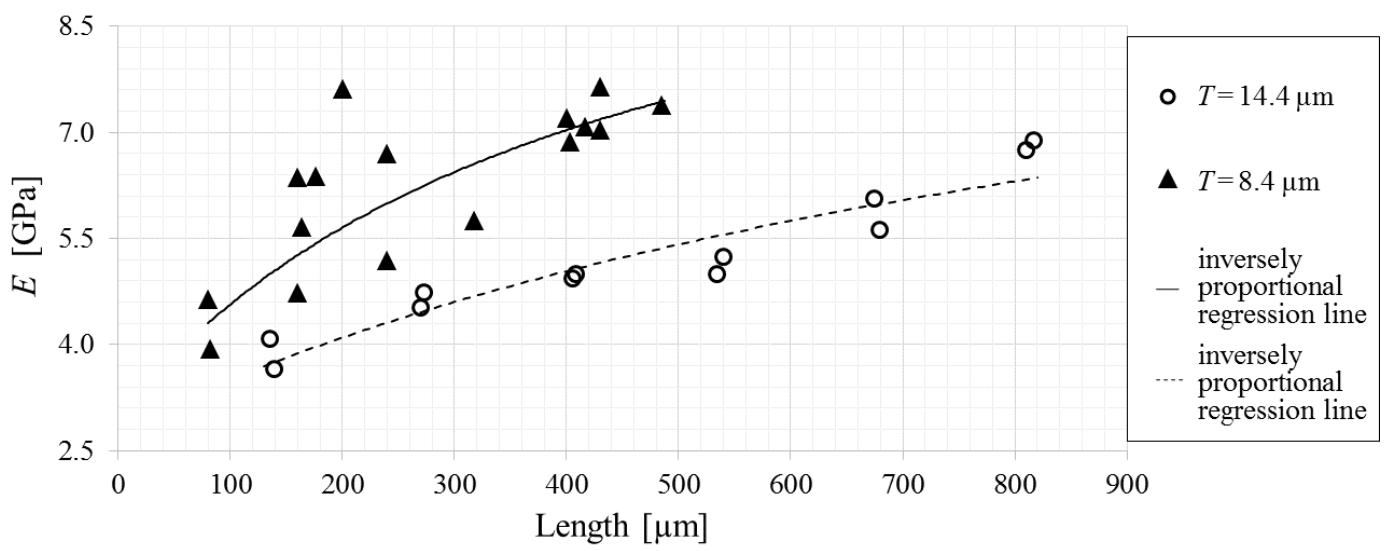

Figure 9: The effect of the length of a beam made of SU-8 on the elastic modulus recorded with the AFM-technique.

Results show that the values of elastic moduli $E$ increase when the thickness of the cantilevers is decreased. The increase of elastic modulus correlates positively with the length $L$ (see Fig. 9) and the values have nearly doubled in the case of the thinnest cantilevers. The size effect, that has been detected here, manifests itself in the slopes of the partial regression lines of the experimental data which show an overall increase with decreasing beam thicknesses. On the basis of general predictions of the material surface theory (GURTIN \& MURDOCH 1975 [18]), regression lines were constructed as inversely proportional functions and independent of the actual thickness. We observe a dependency of $E$ on the length $L$ as well as on the thickness $T$.

\subsection{Model identification}

The discussed experimental results show a very complex behaviour of the real micro-beam. The scale effect is vivid when dimensions of rectangular prism specimen $(L, W, T)$ are changed, and is more pronounced when these dimensions are comparable with an assumed real intrinsic length scale. In this sense, from the point of view of modelling, a 2D or even 3D description should be applied. Additionally, anisotropic length scale effects could be covered in a 2D or 3D description. Nevertheless, we claim that the FEBB model described here is able to model the present size effects with respect to a change of beam lengths and beam thicknesses by finding appropriate combinations of the two additional introduced parameters $\alpha$ and $\ell$, together with the type of fractional derivative (in our case the RIESZ-CAPUTO one). However, similar to the CEBB model, the information about the cross section is only included in the second moment of inertia (cf. Eq. 27). Thus, from the first sight one can expect, that only scale effects between different micro-beam's lengths $(L)$ can be covered by FEBB, but not between different thicknesses or widths $(T$ or $W$ ). Fortunately, such a statement is not true at all. In our observations, a change of both, length and cross sectional area of micro-beams can be covered by FEBB formulation, by means of changing the order of fractional continuum $0<\alpha<1$. In Fig. 10 the comparison of the experimental results (cf. Fig. 9) and the results of the FEBB model are presented. Non-local moduli $E_{\mathrm{NL}}$ have been calculated according to Eq. 35, utilising numerical deflections $w(L, \alpha)$. We observe, that the length scale in our FEBB model should be $\ell=60 \mu \mathrm{m}$, whereas YounG's modulus should be $E=6.9 \mathrm{GPa}$. The effect of a change of cross sections is modelled using a different order of fractional continua, namely: for $T=8.4 \mu \mathrm{m}$ and $W=82 \mu \mathrm{m}$ we have $\alpha=0.8$; and for $T=14.4 \mu \mathrm{m}$ and $W=122 \mu \mathrm{m}$ we have $\alpha=0.4$. It should also be emphasised, that the experimental result, that when the length $L$ of micro-beams is growing, the non-local elastic modulus $E_{\mathrm{NL}}$ converges to a single value. 
This gives evidence to the previously expected behaviour of the FEBB model, that if the dimensions of beams become significantly larger than the length scale $(L, W, T \gg \ell)$, the non-local modulus $E_{\mathrm{NL}}$ converges to the classical elastic modulus $E$.

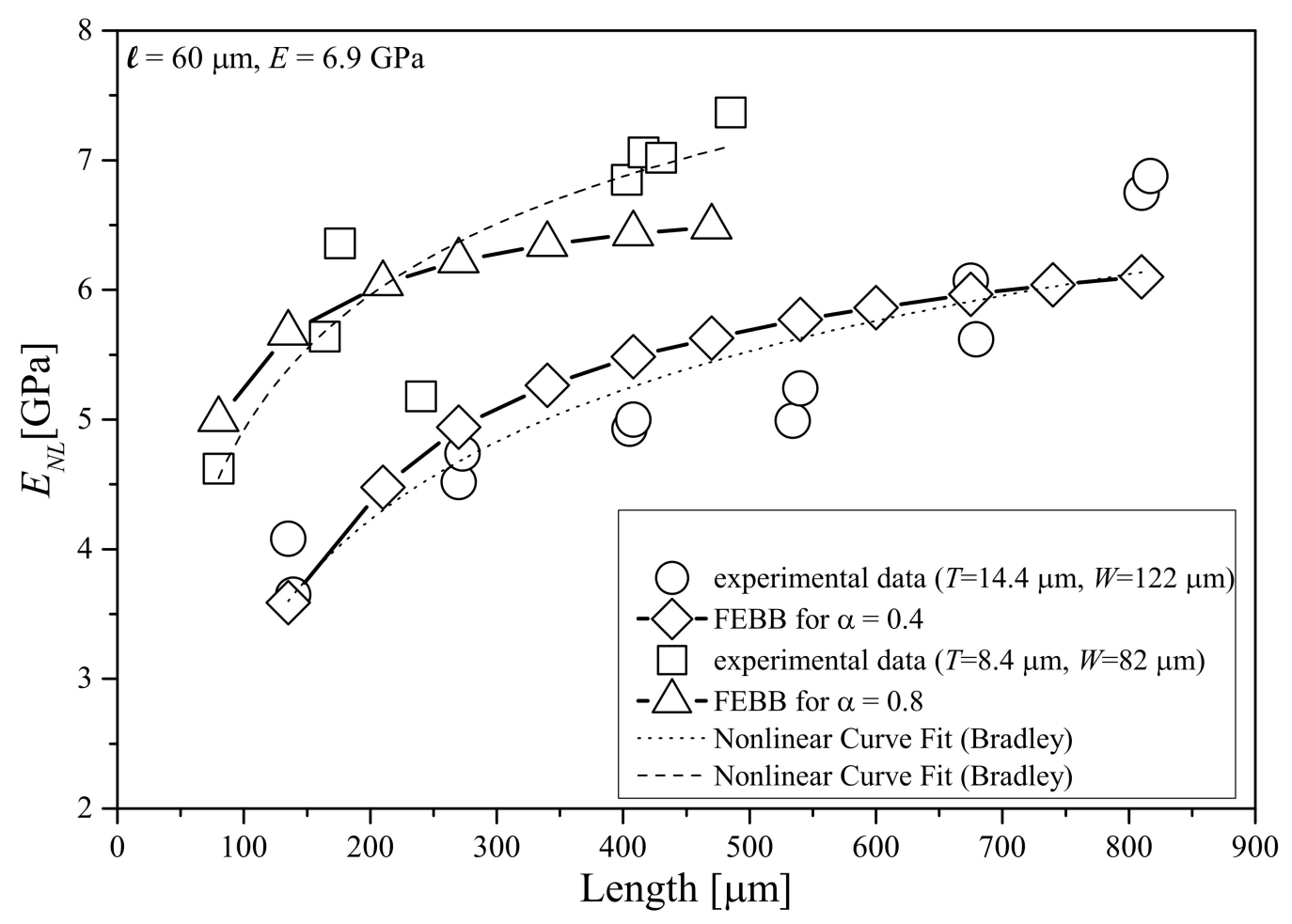

Figure 10: Comparison of the experimental results with the FEBB model.

\section{Conclusions}

In the present work, the non-local fractional Euler-Bernoulli beam theory is formulated as a generalisation of classical EULER-BERNOULLI beams, utilising fractional calculus. The model is implemented using codes developed by the authors and identified based on AFM experiments concerning bending of micro-beams made of the polymer SU-8, where scale effect is severely manifested. The fractional EULER-BERNOULLI beam model introduces two additional parameters $\alpha$ and $\ell$ together with the type of fractional derivative (in the discussed case the RIESZ-CAPUTO one). It is shown, that this new model is able to give, in a qualitative and quantitative manner, good approximation of the revealed experimental results.

\section{Acknowledgement}

The present work is partially supported by (Deutsche Forschungsgemeinschaft) DFG under Grant MU 1752/33-1 and the National Centre for Research and Development (NCBiR) under Grant No. UODDEM-1-203/001. The authors would like to thank the Fraunhofer Institute for Reliability and Microintegration Berlin for sample preparation and PTB-Braunschweig for AFM calibration. 


\section{References}

[1] T.M. Atanackovic and B. Stankovic. Generalized wave equation in nonlocal elasticity. Acta Mechanica, 208(1-2):1-10, 2009.

[2] Asad J.H. Baleanu D. and Petras I. Fractional Bateman-Feshbach Tikochinsky Oscillator. Communications in Theoretical Physics, 61:221-225, 2014.

[3] O.A. Bauchau and J.I. Craig. Structural Analysis with Application to Aerospace Structures. Springer, Dordrecht, Heidelberg, London, New-York, 2009. ISBN 978-90-481-2515-9.

[4] T. Blaszczyk. A numerical solution of a fractional oscillator equation in a non-resisting medium with natural boundary conditions. Romanian Reports In Physics, 67(2), 2015. in print.

[5] T. Blaszczyk and M. Ciesielski. Numerical solution of fractional sturm-liouville equation in integral form. Fractional Calculus and Applied Analysis, 17(2):307-320, 2014.

[6] T. Blaszczyk, J. Leszczynski, and E. Szymanek. Numerical solution of composite left and right fractional caputo derivative models for granular heat flow. Mechanics Research Communications, 48:42-45, 2013.

[7] Cresson J. Greff I. Bourdin, L. and P Inizan. Variational integrator for fractional euler-lagrange equations. Applied Numerical Mathematics, 71:14-23, 2013.

[8] A. Carpinteri. Fractal nature of material microstructure and size effects on apparent mechanical properties. Mechanics of Materials, 18:89-101, 1994.

[9] A. Carpinteri, P. Cornetti, and A. Sapora. A fractional calculus approach to nonlocal elasticity. European Physical Journal Special Topics, 193:193â€“"204, 2011.

[10] C.M. Chong. Experimental investigation and modeling of size effect in elasticity. Dissertation, Hong Kong University of Science and Technology, 2002.

[11] M. Ciesielski and J. LeszczyĹ,ski. Numerical solutions to boundary value problem for anomalous diffusion equation with Riesz-Feller fractional derivative. Journal of Theoretical and Applied Mechanics, 44(2):393-403, 2006.

[12] S. Cuenot, C. Fretigny, S. Demoustier-Champagne, and B. Nysten. Surface tension effect on the mechanical properties of nanomaterials measured by atomic force microscopy. Physical Review B, 69:01-05, 2004.

[13] M. Di Paola, G. Failla, and M. Zingales. Physically-based approach to the mechanics of strong non-local linear elasticity theory. Journal of Elasticity, 97(2):103-130, 2009.

[14] C.S. Drapaca and S. Sivaloganathan. A fractional model of continuum mechanics. Journal of Elasticity, 107:107-123, 2012.

[15] A.C. Eringen. Linear theory of micropolar elasticity. Journal of Mathematics and Mechanics, 15:909-923, 1966.

[16] A.C. Eringen. Nonlocal Continuum field theories. Springer, New York, 2010.

[17] N.A. Fleck, G.M. Müller, M.F. Ashby, and J.W. Hutchinson. Strain gradient plasticity: Theory and experiment. Acta Metallurgica et Materialia, 42(2):475-487, 1994. 
[18] M.E. Gurtin and A.I. Murdoch. A continuum theory of elastic material surfaces. Archive for Rational Mechanics and Analysis, 57(4):291-323, 1975.

[19] A.A. Kilbas, H.M. Srivastava, and J.J. Trujillo. Theory and Applications of Fractional Differential Equations. Elsevier, Amsterdam, 2006.

[20] M. Klimek. Fractional sequential mechanicsâ€"models with symmetric fractional derivative. Czechoslovak Journal of Physics, 51(12):1348â€“1354, 2001.

[21] K.A. Lazopoulos. Non-local continuum mechanics and fractional calculus. Mechanics Research Communications, 33:753â€“757, 2006.

[22] J.S. LeszczyĹ,ski. An introduction to fractional mechanics. Monographs No 198. The Publishing Office of Czestochowa University of Technology, 2011.

[23] H. Lorenz, M. Despont, N. Fahrni, N. LaBianca, P. Renaud, and P. Vettiger. A low-cost negative resist for MEMS. Journal of Micromechanics and Microengineering, 7:121-124, 1997.

[24] Q. Ma and D.R. Clarke. Size dependent hardness of silver single crystals. Journal of Materials Research, 10(4):853-863, 1995.

[25] A.W. McFarland and J.S. Colton. Role of material microstructure in plate stiffness with relevance to microcantilever sensors. Journal of Micromechanics and Microengineering, 15(5):1060-1067, 2005.

[26] R.D. Mindlin and N.N. Eshel. On first strain-gradient theories in linear elasticity. International Journal of Solids and Structures, 4:109-124, 1968.

[27] S. Nikolov, C.-S. Han, and D. Raabe. On the origin of size effects in small-strain elasticity of solid polymers. International Journal of Solids and Structures, 44:1582-1592, 2007.

[28] W. Nowacki. Theory of Micropolar Elasticity. CISM, Udine, 1972.

[29] Z. Odibat. Approximations of fractional integrals and Caputo fractional derivatives. Applied Mathematics and Computation, 178:527â€"533, 2006.

[30] E.C. Oliveira and J.A.T. Machado. A review of definitions for fractional derivatives and integral. Mathematical Problems in Engineering, 2014(Article ID 238459):6 pages, 2014.

[31] J. Peddieson, G.R. Buchanan, and R.P. McNitt. The role of strain gradients in the grain size effect for polycrystals. International Journal of Engineering Science, 41:305-312, 2003.

[32] I. Podlubny. Fractional Differential Equations, volume 198 of Mathematics in Science and Engineering. Academin Press, 1999.

[33] J.-P. Salvetat, G. Andrew, D. Briggs, J.-M. Bonard, R.R. Bacsa, A.J. Kulik, T. Stöckli, N.A. Burnham, and L. Forró. Elastic and shear moduli of single-walled carbon nanotube ropes. Physical Review Letters, 82(2):944-947, 1999.

[34] V.P. Smyshlyaev and N.A. Fleck. The role of strain gradients in the grain size effect for polycrystals. Journal of the Mechanics and Physics of Solids, 44(4):465-495, 1996.

[35] G. Stan, C.V. Ciobanu, P. M. Parthangal, and R.F. Cook. Diameter-dependent radial and tangential elastic moduli of zno nanowires. Nano Letters, 7(12):3691-3697, 2007. 
[36] W. Sumelka. Thermoelasticity in the framework of the fractional continuum mechanics. Journal of Thermal Stresses, 37(6):678â€“706, 2014.

[37] W. Sumelka and T. Blaszczyk. Fractional continua for linear elasticity. Archives of Mechanics, 66(3):147â€"“172, 2014.

[38] W. Sumelka, K. Szajek, and T. Lodygowski. Plane strain and plane stress elasticity under fractional continuum mechanics. Archive of Applied Mechanics, 2014. DOI:10.1007/s00419-0140949-4.

[39] R.A. Toupin. Elastic materils with couple-stress. Archive for Rational Mechanics and Analysis, 11(1):385-414, 1962.

[40] M. Varenberg, I. Etsion, and G. Halperin. Nanoscale fretting wear study by scanning probe microscopy. Tribology Letters, 18(4):493-498, 2005.

[41] L. Vazquez. A fruitful interplay: from nonlocality to fractional calculus. In F.Kh. Abdullaev and V.V. Konotop, editors, Nonlinear Waves: Classical and Quantum Aspects, page 129â€“"133. 2004.

[42] Xu Y. and Agrawal O.P. Models and numerical solutions of generalized oscillator equations. Journal of Vibration and Acoustics, 136:051005-1-051005-7, 2014. 